Nach drei Jahren Zeitschriftenleben den Versuch einer Zwischenbilanz zu machen, ist sicher nicht besonders voreilig, bedenkt man die stürmische Entwicklung, die der Sektor "Rechtsinformatik" in letzter Zeit genommen hat. Wir möchten bei luR diese Bilanz nicht ohne unsere Abonnenten und Leser ziehen und würden uns deswegen über Zuschriften freuen, die uns für die Pläne des Jahres 1989 Orientierungshilfen geben. Besonders interessiert uns, ob der Akzent der Zeitschrift in Zukunft stärker auf den Rechtsproblemen der EDV liegen sollte oder ob eher eine "Anwenderzeitschrift" gewünscht wird, die Hard- und Software als Hilfsmittel des Juristen bei seiner Arbeit ins Auge faßt. Für die konzeptionelle Planung ist es auch wichtig zu wissen, ob unsere Leser eine Verstärkung der „juris-Komponente” wünschen, die dem gewachsenen Rang dieser Datenbank entspricht.

Und schließlich wüßten wir gerne, ob die Verbindung der Zeitschrift mit dem mailbox-gestützten elektronischen Newsletter "Informatik und Recht" intensiviert werden soll. Als kleinen Dank für die Mühe, die Sie sich mit einem Schreiben zu diesen (und anderen, die Zeitschrift betreffenden Fragen) machen, haben wir eine "Überraschungsdiskette” für Sie vorbereitet. Schreiben erreichen den Herausgeber entweder unter der Anschrift: Lehrstuhl für Rechtsinformatik, Universität des Saarlandes, Im Stadtwald, 6600 Saarbrücken, oder in der Mailbox (NUA: 45612133061 ). Abteilung "luR".

Mit allen guten Wünschen für 1989

bin ich

Ihr

\title{
Maximilian Herberger
}

P.S. Im Anzeigenteil dieses Hefts versteckt sich ein weiteres kleines Präsent, das die PC-Arbeit wirksam unterstützt.

\section{Closse}

\section{Auf Messers Schneide}

Ein großer deutscher Computerhersteller (der Name ist der Redaktion bekannt) hat sich, um eines seiner Produkte den Interessenten dauerhaft in Erinnerung zu bringen, etwas ganz besonderes einfallen lassen: Auf der diesjährigen CeBit in Hannover überreichte man auf dem Messestand in der Halle 1 den an einem Geschäftsstellenprogramm für die Justiz interessierten Besuchern ein Werbegeschenk, das zu Spekulationen vielfältigen Anlaß gibt: Ein Klappmesser „SPORTSMAN'S FOLDING LOCK KNIFE“, made in China, mit einer hervorragend geschärften, spitzen, feststellbaren Klinge - Länge zweiundachtzig Millimeter und einem edelholzbesetzten Messinggriff. Auf der linken Seite der Klinge prangt der Name des betreffenden Programms, geätzt.

Was wollte uns der Dichter, pardon, der Computerhersteller, damit sagen?

Empfindet man das Programm als „ätzend“, steht es gar - wie sein eingeätzter Name - auf Messers Schneide?

Führt der Programmeinsatz zu Hauen und Stechen bei den Anwendern, oder möchte man es solchermaBen durchsetzen (Warnung an die Konkurrenz)?

Soll jemand oder etwas über die Klinge springen?

Liegt in dem eher klassischen Design des Messers womöglich eine versteckte Anspielung (und wenn ja, worauf)?
Vielleicht aber ist das Messer für den „Sportsman“ einfach nur ein Hinweis auf Sportsgeist, den man braucht, wenn man mit dem Programm arbeitet, auf letzte Lösungsmöglichkeiten bei "gordischen Programmknoten"?

Möglicherweise hat man auch an etwas Einschneidendes gedacht, an einen großen Schnitt (Rationalisierung?), an die Freizeit für die Anwender und ihre „freizusetzenden“ Kolleg(inn)en, für die ein Sportmesser in Natur und Wildnis wichtig sein mag.

Der Chronist, der als Linkshänder auf die Betrachtung des Namens bei der Benutzung des Messers übrigens verzichten muß, ihn infolgedessen auch dem Schneidobjekt kaum angemessen präsentieren könnte, hat eine ganz andere, harmlose Vermutung: Sicher hat ein freundlicher Zulieferer aus dem fernen Osten die schneidigen Instrumente als Zugabe mitgeliefert, und weil auch ein großer Konzern schließlich nichts verkommen läßt, wurde mir nix, UNIX, der Name eingeätzt.

Wean nun aber doch eine Absicht dahintersteckt, dürfen wir uns sicher auf neue Überraschungen im nächsten Jahr gefaßt machen. Vorschlag: eine Hängematte. Zum Ausruhen, falls das Programm „hängt“ ...

Judex Calculans 